\title{
The extreme P-Cygni star HDE 316285
}

\author{
D. John Hillier \\ Department of Physics and Astronomy, University of Pittsburgh, \\ 3941 O'Hara Street, Pittsburgh, PA 15260, USA
}

\author{
Paul A. Crowther \\ University College, London, England
}

Francisco Najarro de la Parra

CSIC, Madrid, Spain

\author{
Alexander W. Fullerton \\ The Johns Hopkins University, Baltimore, USA
}

\begin{abstract}
New line-blanketed models for the P-Cygni star HDE 316285 have been calculated. Representative model atoms for H, He I, He II, Ca I, Ca II, Mg I, $\mathrm{Mg}$ II, Al II, Al III, Fe II, and Fe III are included in the calculations. We confirm the basic parameters derived by Hillier et al. (1998) in an earlier unblanketed study, and also confirm that many of the optical metal lines are produced by continuum fluorescence. The charge exchange reaction $\mathrm{Fe}^{2+}+\mathrm{H} \rightleftharpoons \mathrm{Fe}^{+}+\mathrm{H}+$ modifies the $\mathrm{Fe}$ ionization structure and significantly enhances the strength of the optical Fe II emission lines.
\end{abstract}

\section{Discussion}

HDE 316285 is a Galactic P-Cygni-type supergiant whose spectrum is dominated by H, He I, and Fe II P-Cygni profiles. Emission lines due to N I, N II, [N II], O I, Na I, Mg II, AlıI, Ca II, Si II, Si III, Fe II and [Fe II] can also be identified. From its spectroscopic variability, its evolved status, its similarity to P Cygni, and the presence of a nebula, Hillier et al. (1998) inferred that it is a LBV. Hillier et al. (1998) derived $T_{*}=15 \mathrm{kK}, \log L_{*} / \mathrm{L}_{\odot}=5.44, \dot{M}=2.4 \times 10^{-4} \mathrm{M}_{\odot} \mathrm{yr}^{-1}$, $v_{\infty}=410 \mathrm{~km} \mathrm{~s}^{-1}, E_{B-V}=1.81 \mathrm{mag}$, and $\mathrm{N}(\mathrm{H}) / \mathrm{N}(\mathrm{He}) \simeq 1.5$ via an unblanketed model analysis.

In order to understand the nature and evolutionary status of HDE 316285 more fully, we have initiated a study of its spectrum using the non-LTE blanketed models of Hillier \& Miller (1998). In our calculations representative model ions for $\mathrm{H}, \mathrm{He}$ I, He II, Ca I, Ca II, Mg I, Mg II, Al II, Al III, Fe II, and Fe III were included. 1,300 atomic levels (450 super levels) and 9,000 bound-bound transitions are allowed for in our calculations. These calculations confirm the basic parameters previously derived by Hillier et al. (1998) although the preliminary results suggest that luminosity is somewhat higher (up to 50\%). The calcula- 

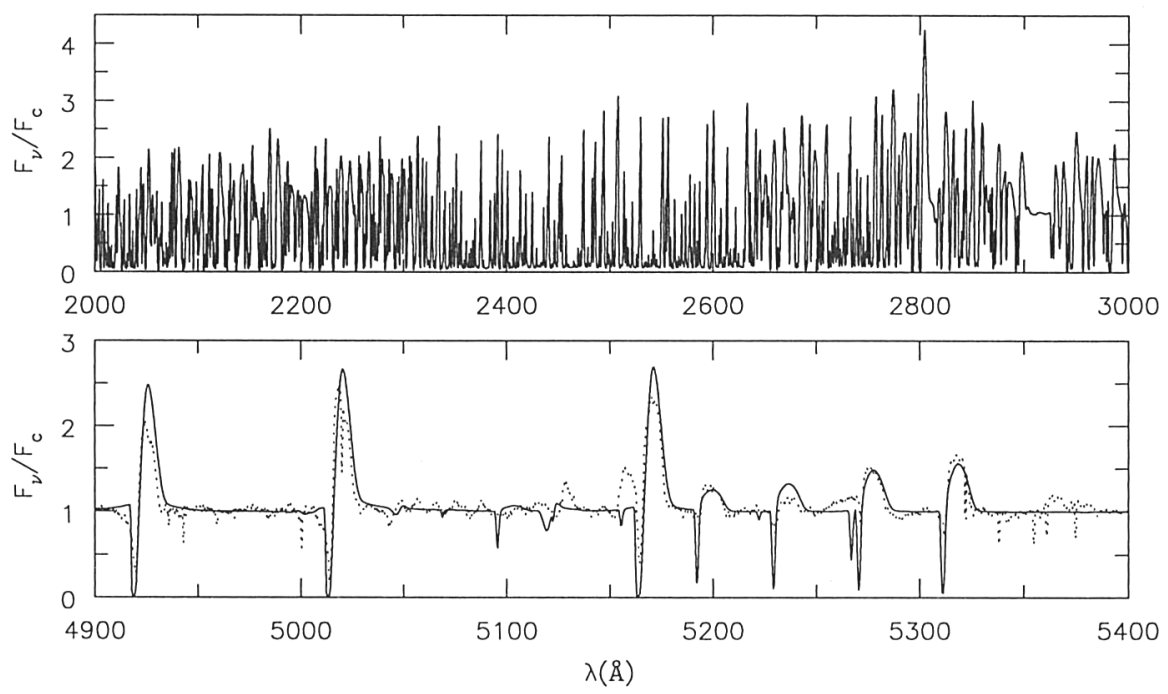

Figure 1. Normalized theoretical and observed spectrum (dots; optical only) of HDE 316285. In the UV, emission, absorption, and P-Cygni emission lines of Fe II completely mask the continuum - indeed the true continuum is only seen in a small band near $2920 \AA$. In the optical, numerous Fe II P-Cygni profiles (e.g., M42 $\lambda \lambda$ 4924, 5018, 5169, M49) are seen.

tions also confirm that many of the emission lines arising from low ionization species (e.g., $\mathrm{Mg}$ II, $\mathrm{Al}$ II) are produced by continuum fluorescence.

One of the striking aspects of the spectrum of HDE 316285 is the strong optical Fe II emission lines. Initial model calculations showed that it was only possible to produce the strong emission profiles if the iron solar mass-fraction was more than four times solar, a value inconsistent with the metallicity deduced from other species (e.g., $\mathrm{Mg}$ ).

In the wind of LBVs there is a significant neutral $\mathrm{H}$ component (e.g., Najarro et al. 1997) hence it is important to allow for charge exchange reactions. Inclusion of the charge exchange reaction $\mathrm{Fe}^{2+}+\mathrm{H} \rightleftharpoons \mathrm{Fe}^{+}+\mathrm{H}^{+}$(Neufeld and Dalgarno 1987) is found to have a major influence on the Fe ionization structure, and significantly enhances the Fe II line strengths (Fig. 1). With the above reaction included, the deduced Fe mass fraction is only twice solar.

\section{References}

Hillier, D.J., Crowther, P.A., Najarro, F., Fullerton, A.W. 1998, A\&A 340, 483

Hillier, D.J., Miller, D.L. 1998, ApJ 496, 407

Najarro, F., Hillier, D. J., Stahl, O. 1997, A\&A 326, 1117

Neufeld, D.A., Dalgarno, A. 1987, Phys. Rev. A. 35, 3142 